Communications in Physics, Vol. 31, No. 2 (2021), pp. 211-217

DOI:10.15625/0868-3166/15382

\title{
SIMULATION OF ANGLE-INSENSITIVE MICROWAVE METAMATERIAL ABSORBERS OPERATING AT TRANSMISSION MODE
}

\author{
TRAN VAN HUYNH ${ }^{1,2,3}$, VU DINH LAM ${ }^{1,2}$ AND NGUYEN THANH TUNG ${ }^{1,2, \dagger}$ \\ ${ }^{1}$ Institute of Materials Science, Vietnam Academy of Science and Technology, \\ 18 Hoang Quoc Viet, Cau Giay, Hanoi, Vietnam \\ ${ }^{2}$ Graduate University of Science and Technology, Vietnam Academy of Science and Technology, \\ 18 Hoang Quoc Viet, Cau Giay, Hanoi, Vietnam \\ ${ }^{3}$ Department of Basic Sciences, University of Fire Prevention and Fighting, Vietnam \\ E-mail: ${ }^{\dagger}$ tungnt@ims.vast.ac.vn
}

Received 15 August 2020

Accepted for publication 29 October 2020

Published 05 January 2020

\begin{abstract}
Metamaterial absorbers with the resonator/dielectric/metallic-mirror conventional design are often angle-dependent and completely reflective outside the absorption band. Herein we have proposed a novel solution to achieve an angle-insensitive bidirectional absorber operating at transmission mode using a simple metamaterials system. The proposed system is composed of two symmetric disk-pair layers, called the disk-pair dimer, that exhibited two identical but coupled magnetic resonances at the same frequency. This unique design allows to absorb the electromagnetic energy at the transmission mode, which is rarely achieved in the conventional modes. By optimizing the coupling strength between two disk-pair layers, a total absorptivity could be enhanced up to $90 \%$.
\end{abstract}

Keywords: perfect absorbers; metamaterials; transmission mode.

Classification numbers: 81.05.Xj; 78.67.Pt.

\section{INTRODUCTION}

Independent manipulation of the electric and magnetic responses through structural engineering for a frequency-selective absorption, which is usually not available in natural occurring materials, is an important advance of metamaterials [1-3]. Since the first introduction in 2008, the novel concept of metamaterial absorbers (MMAs) has gained a huge attention and rapidly

(C2021 Vietnam Academy of Science and Technology 
developed not only for fundamental knowledge but also for the desire of using advanced materials in sensing, energy harvesting, and radiation probing based applications [4-6]. Generally, the interactions of light with MMAs can be described in terms of transmission, reflection, and absorption. The absorption can be defined as $A(f)=1-R(f)-T(f)$, where $A, R$, and $T$ are the absorption, reflection, and transmission as functions of frequency $f$, respectively [1]. An absorption as high as possible can only be achieved by minimizing $R(f)$ and $T(f)$ at the same time. A simple design, which is composed of a three-functional-layer structure, has been proposed for MMAs to simultaneously satisfy both above-mentioned requirements. The structure usually includes periodically-arrayed metallic resonators on top, a dielectric spacer in the middle layer, and a metallic ground plane on the back. First, to diminish the reflection, the effective impedance of metallic resonators is matched to that of the free space through tuning the geometrical parameters of the top resonators. Meanwhile, the metallic ground plane is used as a electromagnetic mirror to quench all incoming waves, resulting in a zero transmission. The absorbed energy is mainly attributed to the dielectric loss generated by the induced coupling between the resonators and the mirror [7-9]. The simplification of this structure in fabrication and characterization makes it most commonly used for existing MMAs in the current literature so far.

Nevertheless, there are still barriers to overcome before the MMAs can be utilized in the real applications. A certain number of studies have been carried out to push the operating frequency of MMAs to either higher regimes, i.e. millimeter waves [10,11], THz [12,13], infrared [14, 15], and visible range [16,17], or lower regimes, i.e. MHz range [18]. Different geometrical structures have been proposed and investigated to improve the polarization and incidence-angle dependence of the MMAs $[16,19]$. Together with attempts to diversify operating frequencies, many efforts have been paid for advancing the MMA functionalities through real-time controlling their absorption band [11] and frequency tunability [20,21]. But the most unpleasant disadvantage of the resonator/dielectric/metallic-mirror motif lies in the fact that it only operates at the reflection mode, sending back all signals at frequencies outside of the absorption range while many applications of absorbers are required to be transparent [22].

Recently, a potential solution has been reported operating at the transmission mode without the metallic mirror [23]. The desired absorption peak is obtained by matching the electric and magnetic resonant losses at the same frequency range while allowing the waves to be transmitted outside the absorption band. Unfortunately, the magnetic and electric resonant frequencies have shown different sensitivities to the incident waves and the resonant superposition lasts only for the incident angles less than 5 degrees. The high absorption behavior hence can only be observed at the normal incident angle, putting an unfortunate limitation on their promising applicability [23]. In order to bridge this gap, we propose a disk-pair (DP) dimer that can exhibit an angle-insensitive bidirectional high absorptivity at transmission mode. The total absorptivity can be achieved up to $90 \%$, resulting in an effective approach to the quasi-omnidirectional frequency-selective absorber. The scattering and dispersive parameters are simulated to get insight into the underlying physics of the hybridized absorption. Finally, an incident-angle sensitivity analysis is performed to characterize the absorption behavior of the proposed metamaterials.

\section{COMPUTATIONAL METHOD}

A computational unit cell of the DP dimer structure is illustrated in Fig. 1(a). The structure consists of two aligned DPs along the $\boldsymbol{k}$-direction. Each pair contains two identical disks arranged 
symmetrically and separated by a dielectric spacer. In our study, the dielectric material is assumed to be FR4 with a dielectric constant of $4+\mathrm{i} 0.008$. The disk-shaped elements are made of copper for microwave-fabrication compatibility. The thicknesses of copper and FR4 are chosen as 0.036 and $1.2 \mathrm{~mm}$, respectively. The disk diameter is fixed at $16.5 \mathrm{~mm}$ while the periodicity of the unit cell in the x-direction and the $\boldsymbol{y}$-direction is kept at $29 \mathrm{~mm}$. The scattering parameters and field distribution are calculated by using the finite integration simulation technique embedded in CST Microwave Studio [24]. To minimize the number of calculations, the periodic boundary conditions are applied in the $\boldsymbol{x} \boldsymbol{x} \boldsymbol{y}$ plane. The standard retrieval method was utilized to extract the effective permittivity and permeability from simulated transmission and reflection [25].

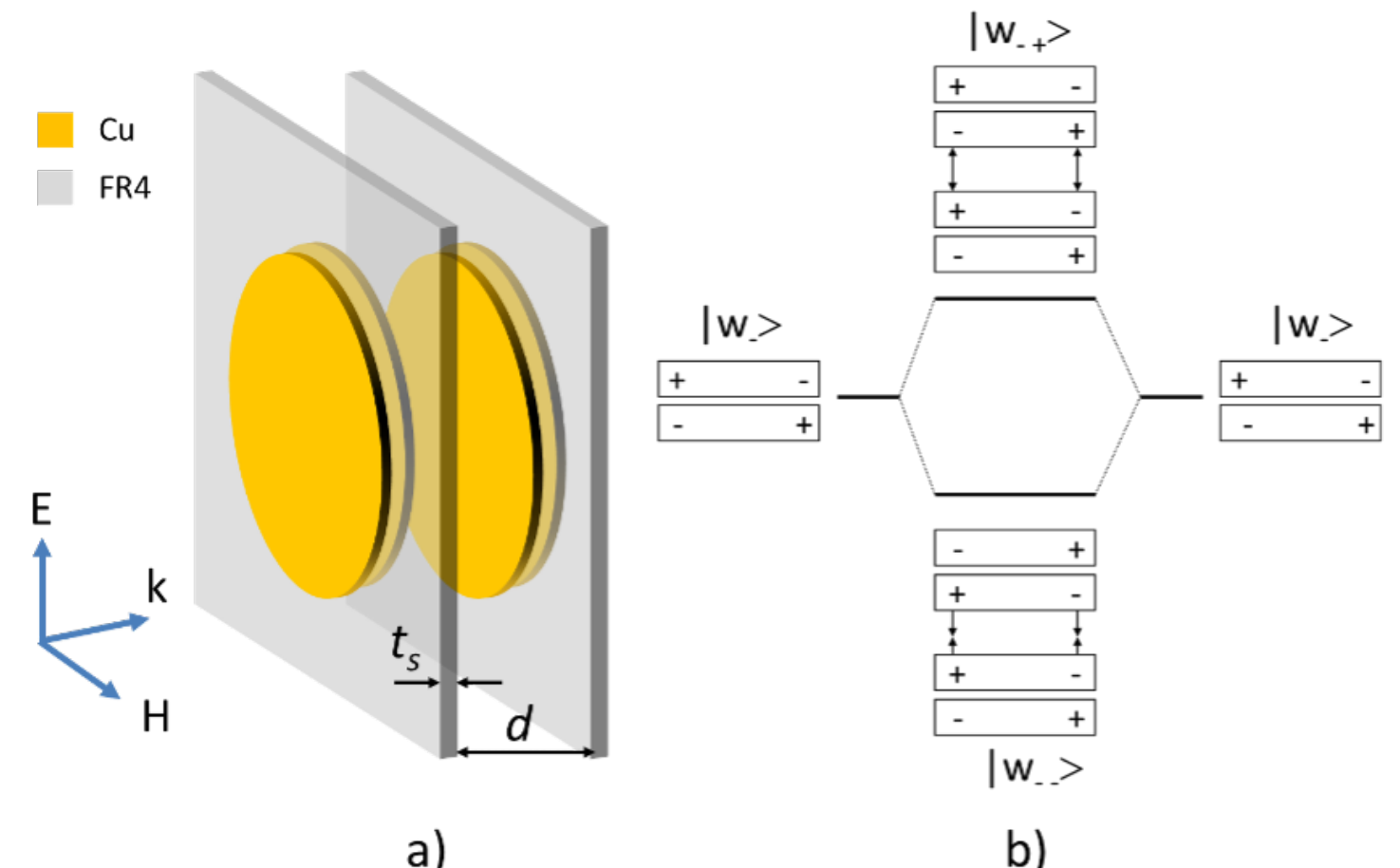

Fig. 1. (a) The computational structure of a disk-pair dimer and its geometrical parameters. (b) The second-hybdrization scheme.

\section{RESULTS AND DISCUSSION}

The electromagnetic behavior of the DP dimer structure can be described by an analog of molecular orbital theory [26]. We first discuss the electromagnetic responses of an isolated single DP monomer metamaterials. Under a normal excitation, the plasmonic response is stimulated in each individual copper disk (the elemental unit). The electromagnetic response, including the magnetic and the electric resonance, of the DP monomer, results from a hybridization of two individual elemental plasmon modes [27]. According to the hybridization scheme, there are two stimulated eigenmodes with opposite current directions. The antisymmetric mode corresponds 
to the magnetically-driven oscillations, $\left(\left|w_{-}\right\rangle\right)$, and the symmetric $\left(\left|w_{+}\right\rangle\right)$mode corresponds to the electrically-driven oscillations. The energy level of each mode depends on the size, shape, and composition of the hybridized system. While the symmetric mode operates as two identical electric dipoles, the antisymmetric mode with antiparallel currents behaves as magnetic resonance. The energetic splitting of two modes is larger if the distance between two disks in a pair $\left(t_{d}\right)$ is smaller [28]. In this study, we focus on the use of the magnetic resonance (mode $\left|w_{-}\right\rangle$) to create the high absorption in metamaterials as shown in Fig. 1(b). The reflection, transmission, and absorption spectra at the magnetic resonant mode $(|w-\rangle)$ of a single DP are shown in the left panel of Fig. 2. The magnetic resonance frequency is determined as about $5 \mathrm{GHz}$, where the transmission and the reflection are strongly fluctuated. A transmission gap appeared at the magnetic resonance reflecting the negative nature of the magnetic permeability that does not allow waves to propagate [29]. The recorded absorption of a single DP is about $45 \%$, in line with previous results [3].
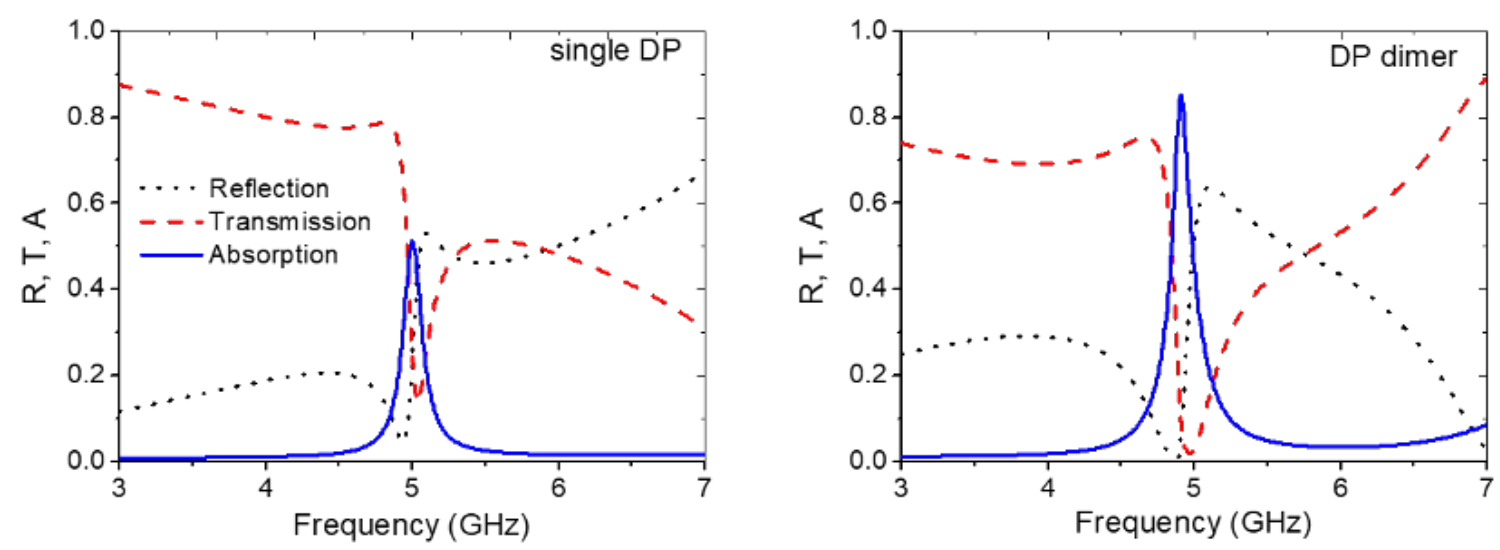

Fig. 2. Computed reflection, transmission, and absorption of the single DP and DP dimer structure with $k=0.5$.

The electromagnetic response of a DP dimer is more interesting. Along with the intrinsic charge interactions within each DP, the additional interactions between two DPs (the back disk of the first DP and the front one of the second DP) also result in Coulomb forces, giving rise to two degenerated magnetic modes corresponding to the second-order antisymmetric $\left|w_{-+}\right\rangle$and the second-order symmetric resonances $\left|w_{--}\right\rangle$[30]. This level of interactions can be illustrated by the second-order hybridization scheme in Fig. 1(b). While the spacer thickness $t_{d}$ responds for the internal coupling between two disks in a DP monomer, the distance $\mathrm{d}$ along the $\boldsymbol{k}$-direction governs the external one between two DPs in a dimer. It has been shown that by properly selecting the ratio $k$ of $d$ to $t_{d}$ one can achieve the best effectiveness of the second-order hybridization for a dual-band negative permeability [30]. Unfortunately, the effect of enhanced absorption and the possibility of using the second-order hybridization for the desired absorption have not been explored in earlier studies. In this work, after performing the optimization procedure a value of 0.5 is chosen for $\mathrm{k}$ to achieve the best absorption performance. The reflection, transmission, and absorption of a DP dimer with $k=0.5$ are presented in the right panel of Fig. 2. It can be seen that the transmission gap corresponding to the negative band of permeability is significantly wider 
due to two degenerated magnetic resonances $\left|w_{--}\right\rangle$and $\left|w_{-+}\right\rangle$. While the transmission gap of degenerated resonances is extended, their corresponding absorption peak is greatly enhanced from $45 \%$ to $90 \%$. The full width at half maximum of the absorption peak also increases from $0.22 \mathrm{GHz}$ for a single DP structure to $0.40 \mathrm{GHz}$ for DP dimer structure. In order to validate the magnetic nature of the observed resonances in the single DP and DP dimer metamaterials, we simulate the distribution of the magnetic and the electric field in each structure at $5 \mathrm{GHz}$. The results are presented in Fig. 3. In the magnetic resonance, the surface currents between two disks in a pair are antiparallel. The currents travel back and forth along the vertical direction of the external electric field and vice versa, resulting in the strong induced electric field at the top and bottom edges of the disks. The antiparallel currents induce a magnetic field between two paired disks along the horizontal direction of the external magnetic field as seen in Fig. 3. Both the single DP and DP dimer show exactly the same electric and magnetic field distribution, confirming their magnetic origin at $5 \mathrm{GHz}$.

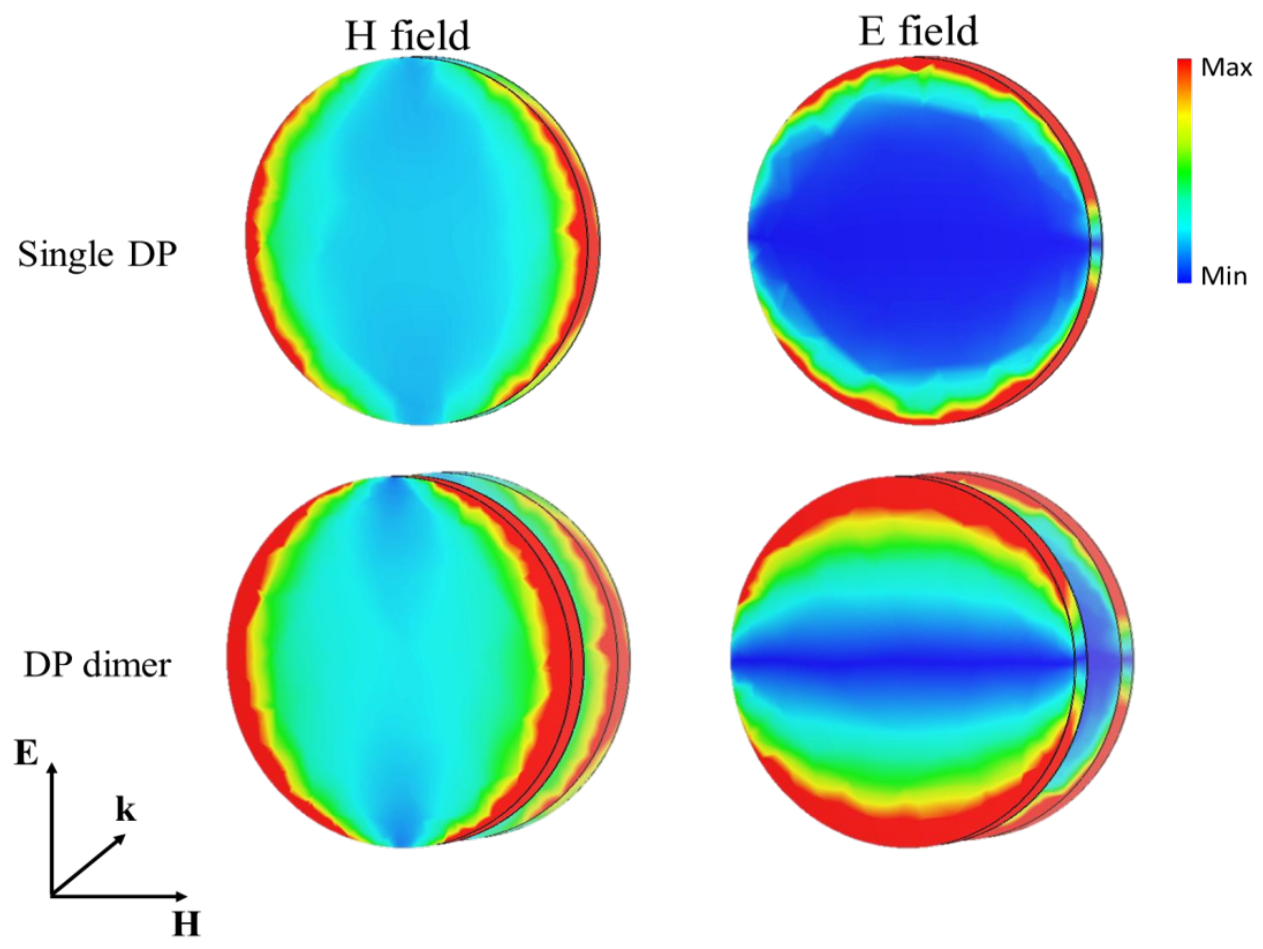

Fig. 3. The simulated E-field and H-field distribution induced in the single DP and DP dimer metamaterials at $5 \mathrm{GHz}$.

As discussed in Section 1, the previously proposed metamaterial absorber operating at the transmission mode is angle-fragile. Since its high absorption strength relies on the overlap of the magnetic and electric resonant frequencies whose sensitivities to the incident waves are strongly different, the resonant superposition lasts only for very small incident angles. The main advantage of our design is that the proposed DP dimer does not only absorb the electromagnetic waves at the 
transmission mode but it also operates at large incident angles. Figure 4 presents our computed results on the absorption intensity of the DP dimer for TE and TM modes at incident angles from 0 to 80 degrees. It is confirmed that the absorption strength is nearly unchanged if the incident angles were up to 70 degrees. It means that the DP dimer can be considered as a 3D quasiomnidirectional absorber. At larger incident angles, the absorption strength rapidly decreases because the external electric and magnetic field become perpendicular to the sample plane for TE and TM mode, respectively. On the other hand, the DP dimer absorber will allow in-plane electromagnetic waves to propagate through.
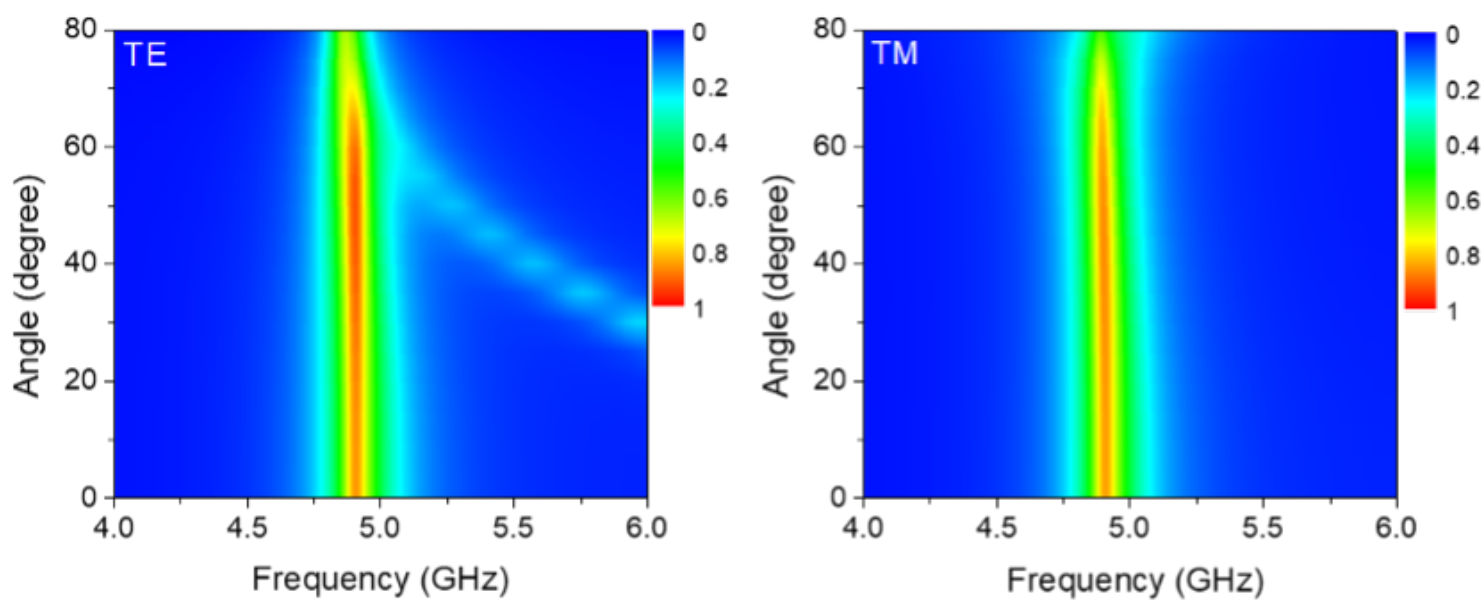

Fig. 4. The computed absorption strength of DP dimer metamaterials according to different incident angles: (left) TE polarization and (right) TM polarization.

\section{CONCLUSIONS}

In summary, we have proposed a simple metamaterial absorber and demonstrated that this absorber can operate at the transmission mode with a wide range of incident angles. The metamaterial absorber consists of two hybridized DP structures. The transmission, reflection and absorption were calculated along with the simulated field distribution to interpret the essence of the absorption peak. The obtained results showed that the high absorption strength of the DP dimer metamaterials originated from a second-order hybridization of magnetic resonances. The insensitivity of absorption intensity to incident angles was examined and this demonstrated its high potential for a wide field of applications. The obtained results can be considered as supplementary valuable information to design advanced metamaterial devices.

\section{ACKNOWLEDGEMENT}

This work is supported by Vietnam National Foundation for Science and Technology Development under grant number FWO.2017.103.01. 


\section{REFERENCES}

[1] N. I. Landy, S. Sajuyigbe, J. J. Mock, D. R. Smith and W. J. Padilla, Phys. Rev. Lett. 100 (2008) 207402

[2] Y. Liu, S. Gu, C. Luo and X. Zhao, Appl. Phys. A 108 (2012) 19.

[3] D. T. Viet, N. V. Van, V. D. Lam and N. T. Tung, Applied Physics Express 8 (2015) 032001.

[4] X. Liu, T. Tyler, T. Starr, A. F. Starr, N. M. Jokerst and W. J. Padilla, Phys. Rev. Lett. 107 (2011) 045901.

[5] N. I. Landy, C. M. Bingham, T. Tyler, N. Jokerst, D. R. Smith and W. J. Padilla, Phys. Rev. B 79 (2009) 125104.

[6] H. Wang and L. Wang,

[7] D. T. Viet, B. S. Tung, L. V. Quynh, N. T. Hien, N. T. Tuan, N. T. Tung, Y. P. Lee and V. D. Lam, Adv. Nat. Sci.: Nanosci. Nanotechnol. 3 (2012) 045014.

[8] D.T.Viet, N.T.Hien, P.V.Tuong, N.QMinh, P.T.Trang, L.N.Le, and V.D.Lam, Perfect absorber metamaterials: Peak, multi-peak and broadband absorption, Opt. Commun. 322 (2014) 209.

[9] N. T. Tung and T. Tanaka, Photonics Nanostructures: Fundam. Appl. 28 (2018) 100.

[10] Han Xiong, Ming-Chun Tang and Jing-Song Hong, Journal of Applied Physics 117 (2015) 154906.

[11] Y.J. Kim, J.S. Hwang, Y.J. Yoo, B.X. Khuyen, X. Chen and Y. P. Lee, Curr. Appl. Phys 17 (2017) 1260

[12] Xiaoyong He, Tunable terahertz graphene metamaterials, CARBON 82 (2015) 229.

[13] W. Wang, K. Wang, Z. Yang and J. Liu, J. Phys. D: Appl. Phys. 50 (2017) 135108

[14] Z. H. Jiang, S. Yun, F. Toor, D. H. Werner, T. S. Mayer, ACS Nano 5 (2011) 4641.

[15] F. Ding, J. Dai, Y. Chen, J. Zhu, Y. Jin and S. I. Bozhevolnyi, Sci. Rep 6 (2016) 39445.

[16] L. Lei, S. Li, H. Huang, K. Tao and P. Xu, Opt. Express 26 (2018) 5686.

[17] W. Wang, Y. Qu, K. Du, S. Bai, J. Tian, M. Pan, H. Ye, M. Qiu and Q. Li, Appl. Phys. Lett. 110 (2017) 101101.

[18] B. X. Khuyen, B. S. Tung, Y. J. Yoo, Y. J. Kim, V. D. Lam, J. G. Yang and Y. P. Lee, Curr. Appl. Phys 16 (2016) 1009.

[19] B. S. Tung, B. X. Khuyen, Y. J. Kim, V. D. Lam, K. W. Kim and Y. P. Lee, Sci. Rep. 7 (2017) 11507

[20] Son Tung Bui, Van Dung Nguyen, Xuan Khuyen Bui, Thanh Tung Nguyen, Peter Lievens, YoungPak Lee and Dinh Lam Vu, Thermally tunable magnetic metamaterials at THz frequencies J. Opt. 15 (2013) 075101.

[21] L. D. Hai, V. D. Qui, N. H. Tung, T. V. Huynh, N. D. Dung, N. T. Binh, L. D. Tuyen and V. D. Lam, Opt. Express 26 (2018) 33253.

[22] J. Ding, B. Arigong, H. Ren, M. Zhou, J. Shao, M. Lu, Y. Chai, Y. Lin and H. Zhang, Scientific reports 4 (2014) 6128.

[23] D. T. Anh, D. T. Viet, P. T. Trang, N. M. Thang, H. Q. Quy, N. V. Hieu, V. D. Lam and N. T. Tung, AIP. Adv 5 (2015) 077119

[24] http://www.cst.com for CST of America, Inc, 492 Old Connecticut Path, Suite 505 Framingham, MA 01701, USA.

[25] Xudong Chen, Tomasz M. Grzegorczyk, Wu Bae-Ian, Joe Pacheco Jr. and Jin Au Kong, Phys. Rev. E 70 (2004) 016608

[26] E. Prodan, C. Radloff, N. J. Halas and P. A. Nordlander, Science 302 (2003) 419

[27] V. T. T. Thuy, D. T. Viet, N. V. Hieu, Y. P. Lee, V. D. Lam and N. T. Tung, Opt. Commun 283 (2010) 4303

[28] B. Kanté, S. N. Burokur, A. Sellier, A. de Lustrac and J.-M. Lourtioz, Phys. Rev. B 79 (2009) 075121

[29] N. T. Tung, D. T. Viet, B. S. Tung, N. V. Hieu, P. Lievens and V. D. Lam, Appl. Phys. Express 5 (2012) 112001

[30] N. T. Tung, B. S. Tung, E. Janssens, P. Lievens and V. D. Lam, Journal of Applied Physics 116 (2014) 083104. 